\title{
Designing Routing and Message-Dependent Deadlock Free Networks on Chips
}

\author{
Srinivasan Murali $^{1}$, Paolo Meloni ${ }^{2}$, Federico Angiolini ${ }^{3}$, David Atienza ${ }^{4},{ }^{6}$, \\ Salvatore Carta $^{5}$, Luca Benini ${ }^{3}$, Giovanni De Micheli ${ }^{4}$, and Luigi Raffo ${ }^{2}$ \\ 1 CSL, Stanford University, Stanford, USA, smurali@stanford.edu \\ 2 DIEE, University of Cagliari, Cagliari, Italy, \\ \{paolo.meloni@diee.unica.it, luigi@diee.unica.it\} \\ 3 DEIS, Univerity of Bologna, Bologna, Italy, \\ \{fangiolini@deis.unibo.it, lbenini@deis.unibo.it\} \\ 4 LSI, EPFL, Lausanne, Switzerland, \\ \{david.atienza, giovanni.demicheli@epfl.ch\} \\ 5 DMI, University of Cagliari, Cagliari, Italy, salvatore@unica.it \\ ${ }^{6}$ DACYA, Complutense University of Madrid (UCM), Madrid, Spain.
}

\begin{abstract}
Networks on Chip (NoC) has emerged as the paradigm for designing scalable communication architecture for Systems on Chips (SoCs). Avoiding the conditions that can lead to deadlocks in the network is critical for using NoCs in real designs. Methods that can lead to deadlock-free operation with minimum power and area overhead are important for designing application-specific NoCs. The deadlocks that can occur in NoCs can be broadly categorized into two classes: routingdependent deadlocks and message-dependent deadlocks. In this work, we present methods to design NoCs that avoid both types of deadlocks. The methods are integrated with the topology synthesis phase of the NoC design flow. We show that by considering the deadlock avoidance issue during topology synthesis, we can obtain a significantly better NoC design than traditional methods, where the deadlock avoidance issue is dealt with separately. Our experiments on several SoC benchmarks show that our proposed scheme provides large reduction in NoC power consumption (an average of 38.5\%) and NoC area (an average of 30.7\%) when compared to traditional approaches.
\end{abstract}

Keywords: Networks on Chips, Systems on Chips, Message-dependent deadlocks, routing-dependent deadlocks, topology, synthesis.

\section{Introduction}

Today's Systems on Chips (SoCs) consist of a large number of computing and storage cores that are interconnected by means of single or multiple layers of buses In order to cope with the large communication demands of such SoCs, a modular, scalable interconnect based on Networks on Chips (NoCs) is needed [1]-[6].

Please use the following format when citing this chapter:

Murali, S., Meloni, P., Angiolini, F., Atienza, D., Carta, S., Benini, L., De Micheli, G. and Raffo, L., 2007, in IFIP International Federation for Information Processing, Volume 249, VLSI-SoC: Research Trends in VLSI and Systems on Chip, eds. De Micheli, G., Mir, S., Reis, R., (Boston: Springer), pp. $337-355$ 
Designing a custom-tailored interconnect that satisfies the performance and design constraints of the $\mathrm{SoC}$ is important to achieve efficient NoC designs [27][32]. A critical, but often neglected issue when designing NoCs is that they have to guarantee deadlock-free operation. If the NoC has no support to either avoid or recover from deadlocks, then correct functionality of the system cannot be guaranteed. This can lead to system crashes and unexpected system behavior, which is clearly unacceptable for SoCs. Designing efficient methods that avoid such a situation with minimum power and area overhead is an important research area in the NoC domain.

The deadlocks that can occur in NoCs can be broadly categorized into two classes: routing-dependent deadlocks and message-dependent deadlocks [33], [7][12]. Routing-dependent deadlocks occur when there is a cyclic dependency of resources created by the packets on the various paths in the network. For regular topologies (such as the mesh, torus), the use of restricted routing functions based on turn models is an effective way to avoid routing-dependent deadlocks [9], [10]. For custom application-specific NoCs, obtaining deadlock-free paths is a bigger challenge [12], [22], [32]. The major focus of this paper is to address this important issue of obtaining routing and message-dependent deadlock-free network operation.

Message-dependent deadlocks occur when interactions and dependencies are created between different message types at network endpoints, when they share resources in the network. Even when the underlying network is designed to be free from routing-dependent deadlocks, the message-level deadlocks can block the network indefinitely, thereby affecting the proper system operation. An example situation where a message-dependent deadlock occurs is presented in Figure 1(a). In this example, two of the cores are masters and two other cores are slaves. In this system, we assume two kinds of messages: request and response. Consider the following situation: Master 1 sends a request to Slave 1 (Req 1$)$, Slave 1 is replying to a previously issued request to Master 1 (Resp 1$)$ and at the same time, Slave 2 sends a response to Master 2 (Resp 2). When requests and responses share the same links, Resp 2 is waiting for link 1 which is used by Req 1 and Resp 1 waits for link 4 used by Resp 2. Meanwhile, Req1 is waiting for Slave 1, the operation of which has been stalled as Resp 1 could not complete. Thus, none of the messages can move ahead, leading to a deadlock situation. An interesting point to note here is that message-level deadlocks can be avoided if the receivers have infinitely large buffering or if they have perfectly ideal operation (consuming all received data instantly), which would avoid queuing of the packets in the network. Obviously, such a solution is not feasible to obtain in practice.

In traditional multi-processor interconnection networks, the most common ways to avoid message-dependent deadlocks are the use of separate logical or physical networks for the different message types [13]-[21]. This would ensure that the different message types do not share the network components, thereby guaranteeing freedom from message-dependent deadlocks. The most common method to achieve separate logical networks is the of use of separate virtual channels for the different message types [13]. For the example design presented 


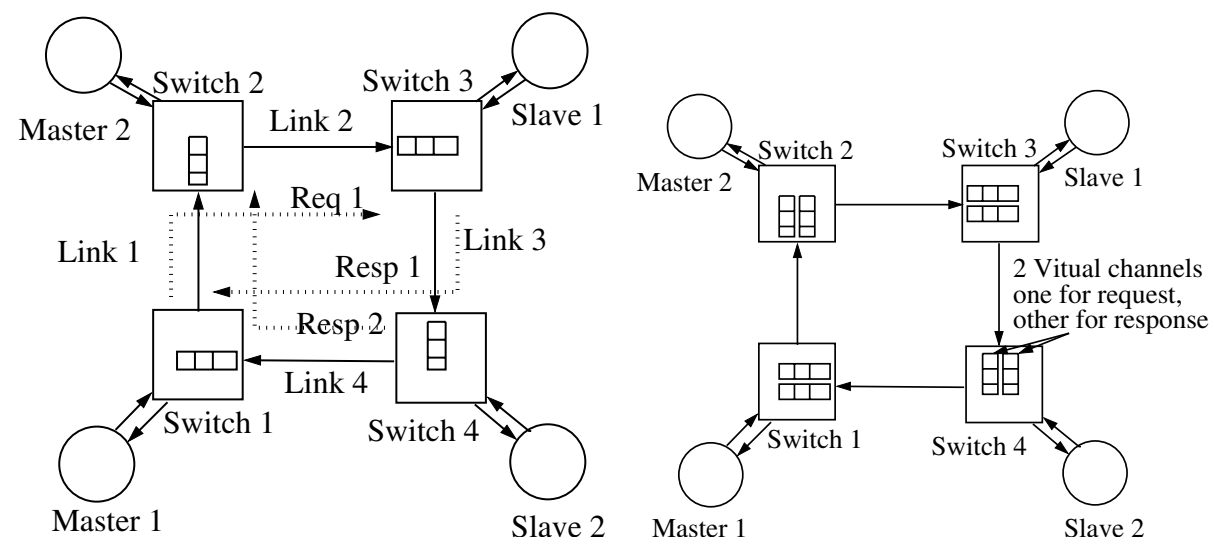

(a) Example of deadlock

(b) Logically separate networks

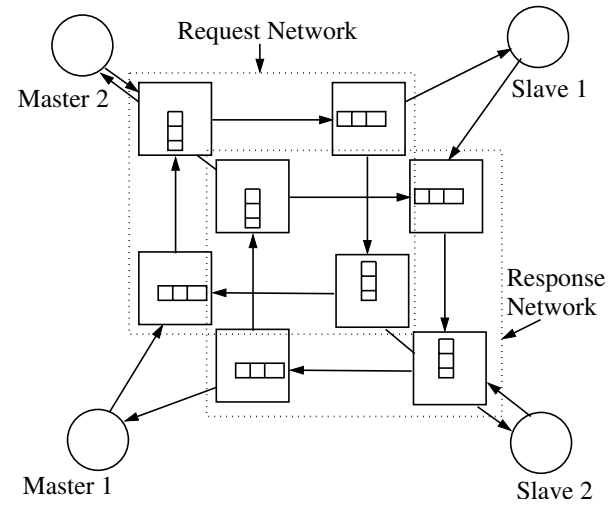

(c) Physically separate networks

Fig. 1. Example of a message-dependent deadlock and traditional methods available to remove it. 
in Figure 1(a), each router input will need two virtual channels: one for the request messages and the other for the response messages (refer Figure 1(b)). This separation of message types is maintained at all the switches in the network. In the case of separate physical networks, the request network is built separately from the response network, an example of which is shown in Figure 1(c). This is the most commonly used solution in complex bus designs such as the STBus from STMicroelectronics [19] and several multi-processor designs [20], [21].

In this work, we show that by mapping the different message types onto different network resources during the topology mapping and synthesis phase, we can achieve much better NoC designs (in terms of power consumption and network area) than traditional approaches. We present a topology synthesis algorithm that specifically considers the message types and ensures the creation of a network that is free from message-dependent deadlocks. We also implement the common methods of deadlock avoidance: having separate virtual channels and having physically separate networks for the message types. For all the schemes, we make the underlying network operation free from routing-dependent deadlocks by integrating existing methods with our topology synthesis process [12]. We perform experiments on several SoC designs, which show that our proposed scheme provides large reduction in the NoC power consumption (an average of $38.5 \%$ ) and area (an average of $30.7 \%$ ) when compared to the traditional approaches.

\section{Previous Work}

The motivation for the use of NoCs has been established in several works [1]-[6]. The use of turn models to avoid deadlocks in mesh and torus networks has been presented in [10]. There has been a large body of work that have focused on developing routing-dependent deadlock-free operation for interconnection networks [9]-[12]. Several other works exist in the area of recovering from deadlocks in networks [7], [8].

The design of application specific NoCs has been explored in several works [24]-[30]. None of these works address the issue of message-level deadlock avoidance, which is critical for proper system operation. Avoiding routing-dependent deadlocks for mesh topologies has been considered in [24]. Avoiding routing deadlocks for custom NoC topologies have been presented in [22], [31].

The use of logically separated networks to avoid message-dependent deadlocks has been utilized in several industrial multi-processors, such as [14]-[17]. The use of physically separated networks to remove message-dependent deadlocks is used in many designs, such as [20], [21]. In [5], message-level deadlock freedom is achieved by a different mechanism than using logically or physically separated networks. It utilizes an end-to-end flow control scheme, which ensures that messages are sent from the sender only when the receiver has enough buffering resources to store them. This is coupled together with a network design that uses time division multiplexing to divide the network resources among the various communicating elements, providing guaranteed throughput to con- 
nections. This leads to buffering free network for such connections and removal from message-level deadlocks. The deadlock avoidance mechanism using their protocol is presented in [23]. As we target general NoC designs that need not support such end-to-end flow control mechanisms, we do not compare such a scheme with our method presented here.

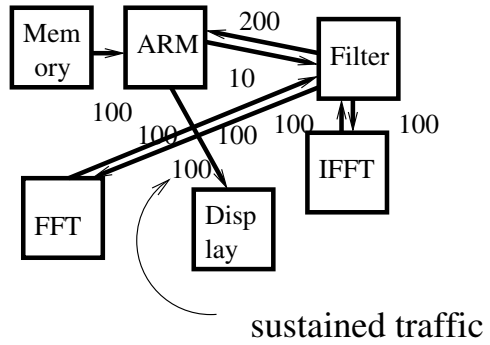

rates

Fig. 2. Example filter application Partition 1

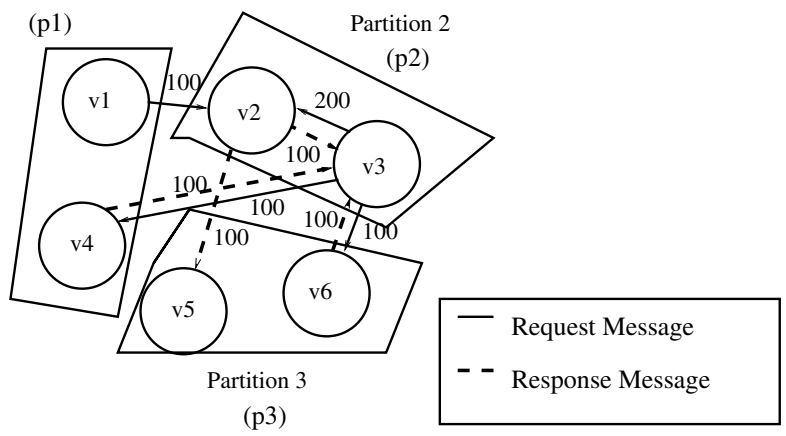

(a) Min-cut partitions

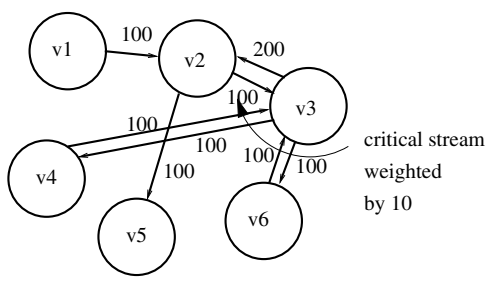

Fig. 3. Core graph with sustained rates and critical streams

Fig. 4. Algorithm Examples

\section{Topology Synthesis with Deadlock Freedom}

We implement our routing and message-dependent deadlock-free path selection routine as a plug-in to an established NoC topology synthesis flow.

\subsection{Topology Synthesis Process}

We assume that the application kernels are parallelized and mapped onto different processors and hardware cores using existing tools, as done in earlier works 
[24]-[32]. The communication traffic flow between the various cores is represented by a core graph, which is taken as the input to the topology synthesis flow. The core graph for a small filter example (Figure 2) is shown in Figure 3. The edges of the core graph are annotated with the sustained rate of traffic flow, multiplied by the criticality level of the flow, as done in [26].

Before presenting the path selection routine, we first present the basic topology synthesis flow. In the topology synthesis procedure (Algorithm 1), we synthesize several topologies: starting from a topology where all the cores are connected to a single switch to a topology where each core is connected to a separate switch. For the chosen switch count, the input core graph is partitioned into those many min-cut partitions (refer to step 2 of Algorithm 1). At this point, the communication traffic flows within a partition has been resolved.

The reason for synthesizing these many topologies is that it cannot be predicted beforehand as to whether a design with few bigger switches can be more power efficient than a design with more smaller switches. A larger switch has more power consumption than a smaller switch to support the same traffic. This is because, a larger switch has a bigger crossbar and arbiter. On the other hand, in a design with many smaller switches, the packets may need to travel more hops to reach the destination. Thus the total switching activity will be higher than a design with fewer hops, which can lead to higher power consumption.

The partitioning is done in such a way that the edges of the graph that are cut between the partitions have lower weights than the edges that are within a partition and the number of vertices assigned to each partition is almost the same. Thus, those traffic flows with large bandwidth requirements or higher criticality level are assigned to the same partition and hence use the same switch for communication. Hence, the power consumption and the hop-delay for such flows will be smaller than the other flows that cross the partitions.

Now, we integrate in the main flow the core contribution of this work (in step 4 of Algorithm 1), i.e. an algorithm (PATH_COMPUTE) that maps the communication flows to physical paths while guaranteeing deadlock freedom. This algorithm is explained in detail in the following paragraphs. Once the paths for a topology are selected, Algorithm 1 resumes, where the design area, power consumption and wire-length for the topologies are obtained. Then, the topology that best optimizes the user objectives and satisfies all the design constraints is chosen. The topology synthesis flow, without considering freedom from message level deadlocks, has been presented by us in detail in [32].

In the following subsections, we explain the path selection mechanism (Algorithm 2) that guarantees routing and message-dependent deadlock-free operation of the NoC.

\subsection{Avoiding Routing Dependent Deadlocks}

When the PATH_COMPUTE procedure is invoked, the number of switches in the $\mathrm{NoC}$ and their connectivity with the cores has already been determined (cores in the same partition share the same switch). The procedure is used to 


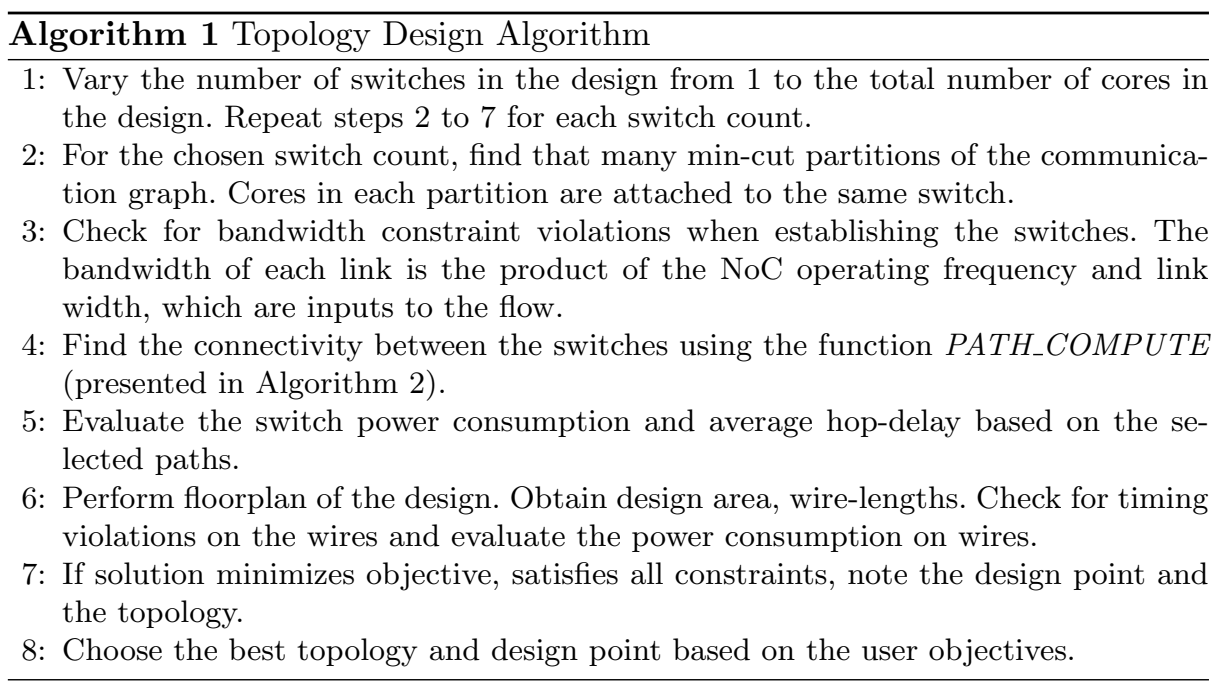

connect the different switches together and find paths for the traffic that flows across the partitions.

In the first step of the procedure, we build a complete graph, with each vertex in the graph representing a switch in the network.

In [12], the authors present a scheme for removing deadlocks in general networks. The approach is also utilized in [22] for removing routing-dependent deadlocks in NoCs. The approach removes routing-dependent deadlocks by prohibiting certain turns for the packets, thereby avoiding cycles in the network. In the next step of the PATH_COMPUTE algorithm, we invoke the BLOCK_TURNS procedure (Algorithm 3) to remove turns in the logical graph to avoid deadlocks. When we compute paths later in the PATH_COMPUTE procedure, we only use those turns that have not been blocked by the BLOCK_TURNS procedure.

\subsection{Avoiding Message-Dependent Deadlocks}

In the next step (step 3) of the PATH_COMPUTE procedure, the flows are ordered in decreasing rate requirements, such that bigger flows are assigned first. The heuristic of assigning bigger flows first has been shown to provide better results (such as lower power consumption and more easily satisfying bandwidth constraints) in several earlier works [25], [31]. Then, for each flow in order, we first evaluate the message type of the flow (step 4 of Algorithm 2). The message types can either be fed explicitly by the user, or can be implicitly considered by the tool. As an example for implicitly considering the type, in shared memory systems, all the traffic flows that originate from processors and terminate into memory devices are of request type. While those that originate from the memories and terminate in the processors are of response type. Note that in shared memory systems, all inter-processor communication occur through the memory 
devices. Note that, if the connection between any pair of cores constitutes multiple message types, then each message type needs to be treated as a separate traffic flow.

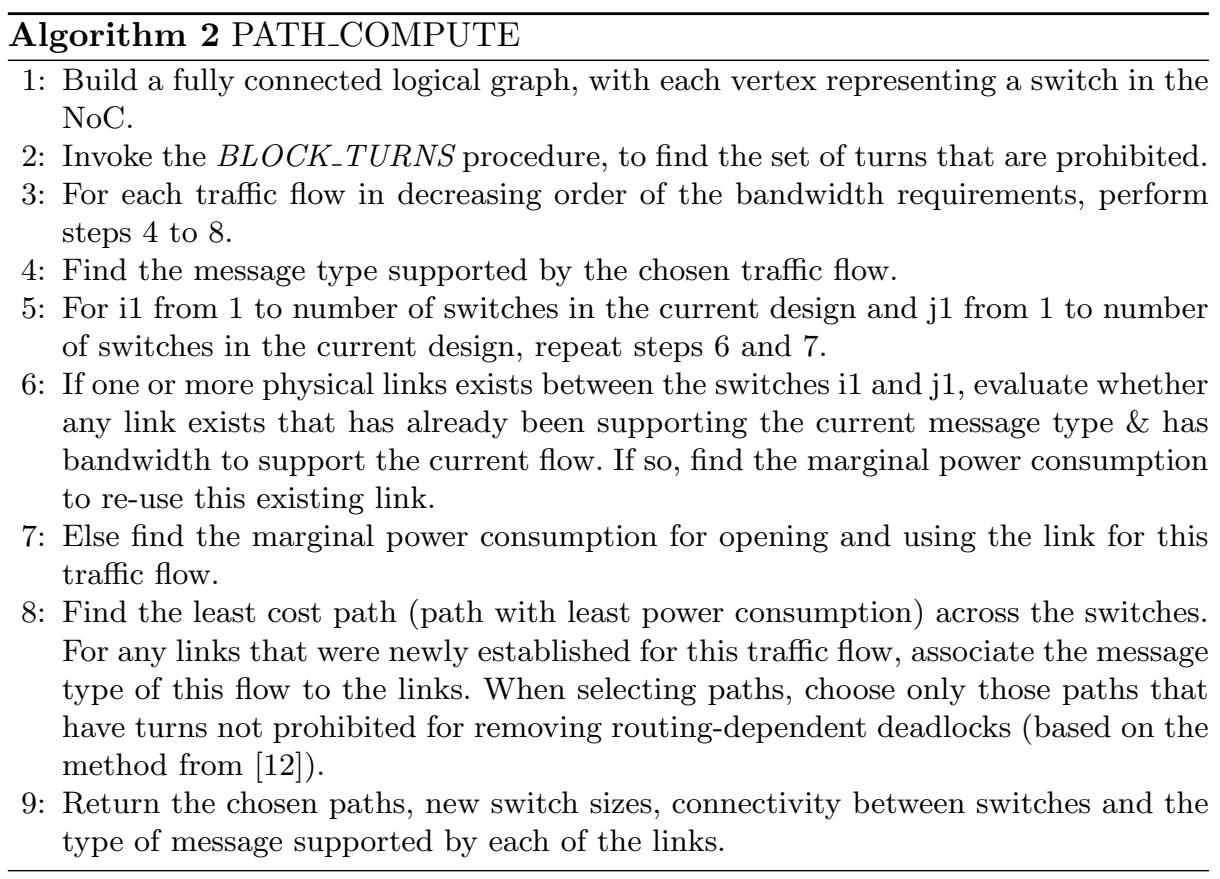

Next, we evaluate the amount of power that will be dissipated across each of the switches, if the traffic for the chosen flow uses that switch. This power dissipation value on each switch depends on the size of the switch, the amount of traffic already routed on the switch and the frequency of operation. It also depends on how the switch is reached (from which other switch) and whether an already existing physical channel will be used to reach the switch or a new physical channel will have to be opened. The last information is needed, because opening a new physical channel increases the switch sizes and hence the power consumption of this flow and others that are routed through the switch.

In our NoC architecture, we permit the instantiation of multiple physical links between any two switches. When finding whether a switch is reachable from another switch for the current traffic flow, we evaluate whether any physical links between the switches have already been established. If so, we see the message type of the traffic flows that have already been routed on the links. From the set of established links, we choose a link that supports the same message type as the current traffic flow and has enough bandwidth available to support the current flow. If no such link is available between the switches, we evaluate the cost of opening up a physical link for the current traffic flow. 
The process of evaluating the power consumption for the current traffic flow is repeated for all pairs of switches. Finally (in step 8 of Algorithm 2), the set of links from the source to destination of the flow that has the least power consumption is chosen. When choosing the paths, only those paths that do not use any turns blocked by the BLOCK_TURNS procedure is considered. Now physical connections are actually established on the chosen path and the message type of the current flow is assigned to the links that have been used for the flow.

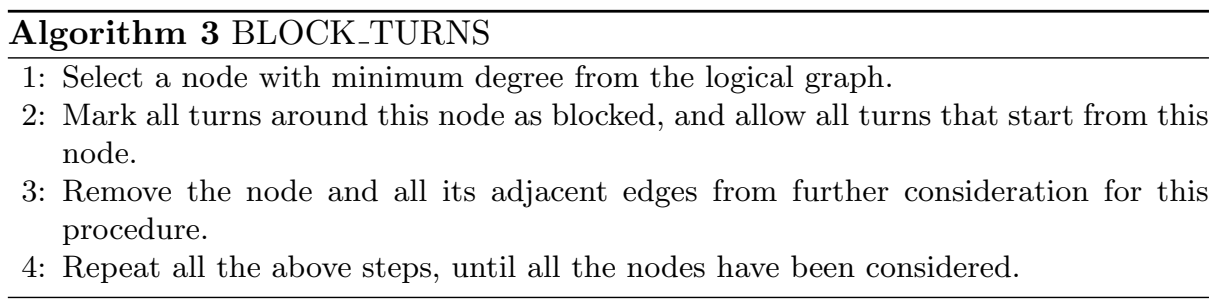

Example 1. Let us consider the example from Figure 4(a). The input core graph has been partitioned into 4 partitions. We assume 2 different message types: request and response for the various traffic flows. Each partition $p_{i}$ corresponds to the cores attached to the same switch. Let us consider routing the flow with a bandwidth value of $100 \mathrm{MB} / \mathrm{S}$ between the vertices $v 1$ and $v 2$, across the partitions $p 1$ and $p 2$. The traffic flow is of the message type request. Initially no physical paths have been established across any of the switches. If we have to route the flow across a link between any two switches, we have to first establish the link. The cost of routing the flow across any pair of switches is obtained. We annotate the edges between the switches by the cost (marginal increase in power consumption) of sending the traffic flow through the switches (Figure 4(b)). The cost on the edges from $p 2$ are different from the others due to the difference in initial traffic rates within p 2 when compared to the other switches. This is because, the switch $p 2$ has to support flows between the vertices v2 and v3 within the partition. The least cost path for the flow, which is across switches $p 1$ and $p 2$ is chosen. Now we have actually established a physical path and a link between these switches. We associate the message type request for this particular link. This is considered when routing the other flows and only those traffic flows that are of request type can use this particular physical link. We also note the size and switching activity of these switches that have changed due to the routing of the current flow.

\section{Experimental Platform}

We have built accurate analytical models for calculating the power consumption, area and delay of the $\times$ pipes network components [36]. 

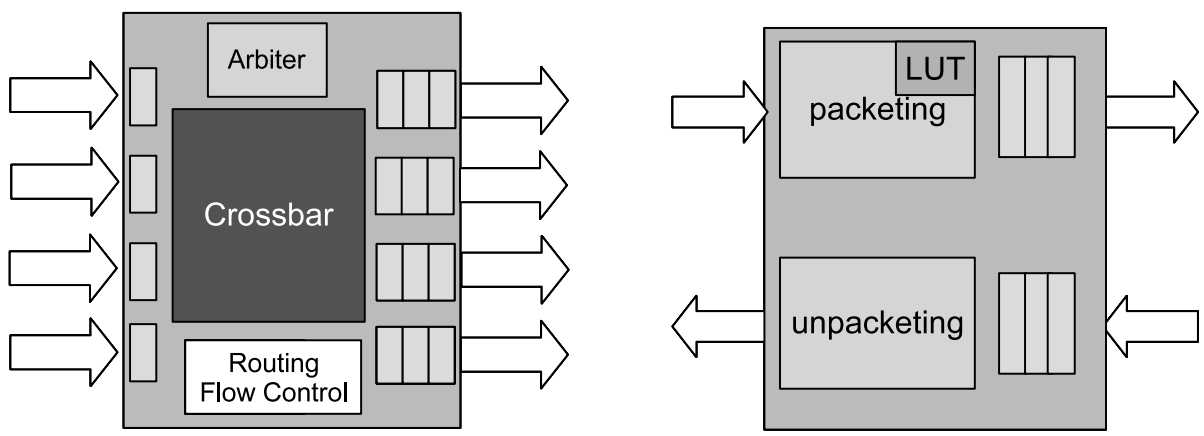

(a)

(b)

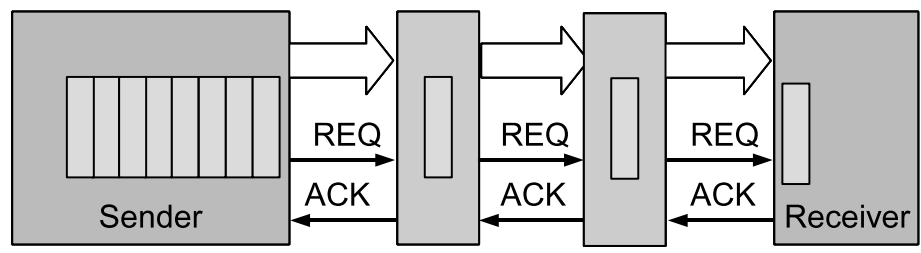

(c)

Fig. 5. $\times$ pipes building blocks: (a) switch, (b) NI, (c) link

\subsection{Architectural Overview}

The $\times$ pipes NoC [36], [37] library consists of a set of parameterizable soft-macros for the network components (Figure 5). The NoC is instantiated by deploying a set of components in an arbitrary topology and by configuring them. There are three main components in the $\times$ pipes library: switches, Network Interfaces (NIs) and links.

The backbone of the NoC is composed of switches, whose main function is to route packets from sources to destinations. Arbitrary switch connectivity is possible, allowing for implementation of any topology. Switches provide buffering resources to lower congestion and improve performance. In $\times$ pipes, output buffering scheme is utilized, where FIFOs are present on each output port of the switch. Switches also handle flow control [38] issues of the NoC, which resolves the conflicts among the packets that request access to the same physical links. The $\times$ pipes architecture supports the use of different flow control strategies, such as the ACK/NACK and STALL/GO protocols. For the experiments performed in this paper, we use the ACK/NACK protocol for flow control.

An NI is needed to connect each IP core to the NoC. NIs convert transaction requests/responses into packets and vice versa. Packets are then split into a sequence of smaller units, referred to as fits (FLow control unITS). In $\times$ pipes, two separate NIs are defined, an initiator and a target one, respectively associated to system masters and system slaves. A master/slave device will require an NI of each type to be attached to it. The interface among IP cores and NIs is 


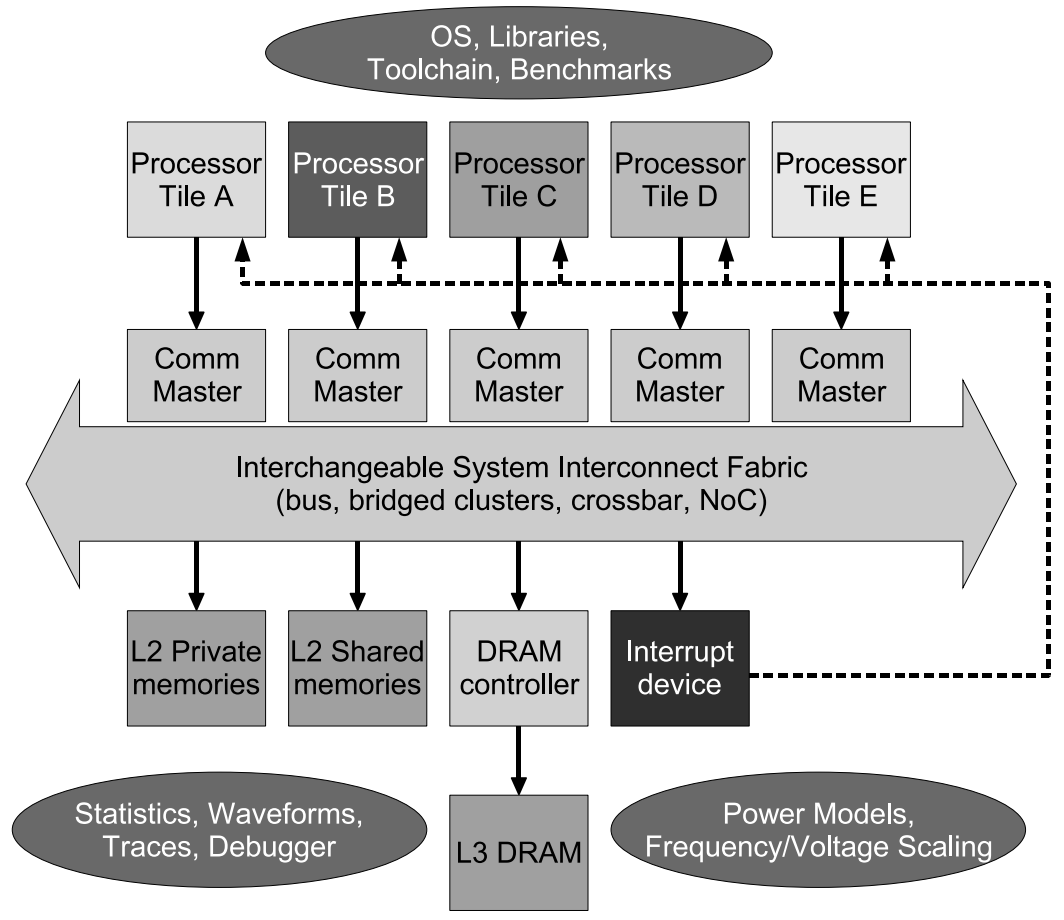

Fig. 6. The MPARM SystemC virtual platform

point-to-point and follows the OCP 2.0 [39] protocol, guaranteeing maximum re-usability. We use source based routing scheme, where each NI has a look-up table to specify the path that packets will follow in the network to reach their destination. Two different clock signals can be attached to NIs: one to drive the NI front-end (OCP interface), the other to drive the NI back-end ( $\times$ pipes interface). The $\times$ pipes clock frequency must be an integer multiple of the OCP one. This arrangement allows the NoC to run at a fast clock even though some or all of the attached IP cores are slower, which is crucial to keep transaction latency low. Since each IP core can run at a different divider of the $\times$ pipes frequency, mixed-clock platforms are possible.

To get accurate simulation in a flexible environment, we integrate the NoC in MPARM (Figure 6). MPARM allows for accurate injection of functional traffic patterns as generated by real IP cores (processors, DMA engines, etc.) during a benchmark run. Further, it provides facilities for debugging, statistics collection and tracing.

\subsection{Area and Power Models}

To get an accurate estimate of these parameters, the place\&route of the components is performed using Cadence SoC Encounter [35]. From the layout-level 


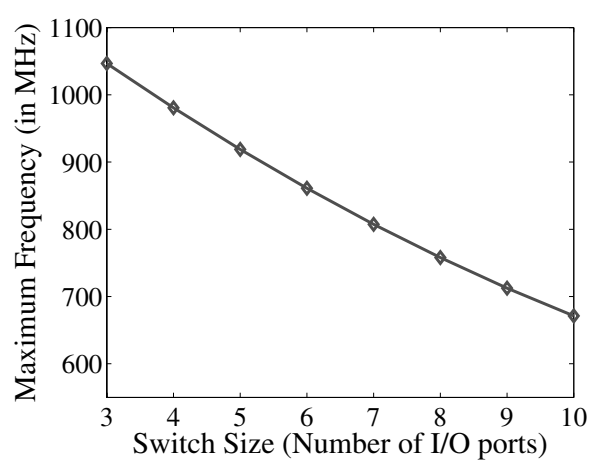

Fig. 7. Maximum frequency variation with switch size

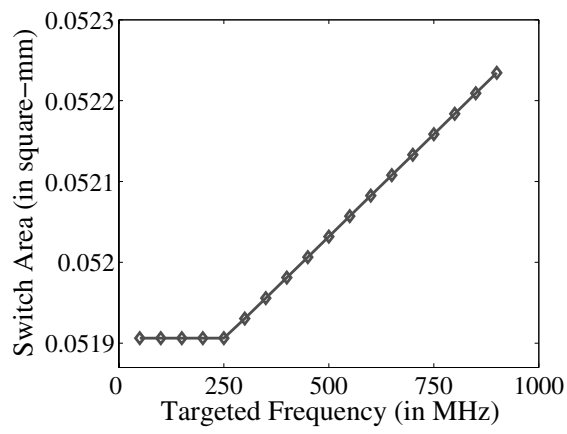

(a) Impact on switch area

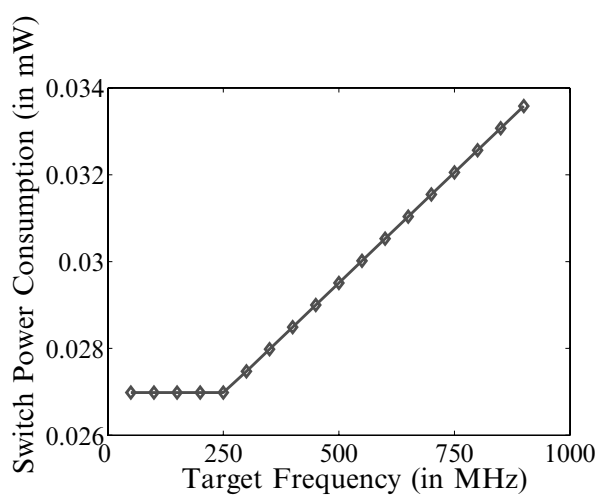

(b) Impact on switch energy

Fig. 8. Impact of frequency on the area and energy of a $5 \times 5$ switch, for $0.13 \mu m$ technology

implementations, the back-annotated accurate wire capacitances and resistances are obtained, with a $0.13 \mu \mathrm{m}$ technology library. The switching activity in the network components is varied by injecting functional traffic. The capacitance, resistance and the switching activity report are combined to estimate power consumption using Synopsys PrimePower [34].

A large number of implementation runs were performed, varying several parameters, such as the number of input, output ports, link-width and the amount of switching activity for the NoC switches. When the size of a NoC switch increases, the size of the arbiter and the crossbar matrix inside the switch also increases, thereby increasing the critical path of the switch. To have accurate delay estimates of the switches, we model the maximum frequency that can be supported by the switches, as a function of the switch size. An example set of values are presented in Figure 7.

We used linear regression to build analytical models for the area and power consumption of the components as a function of these parameters. Due to the intrinsic modularity and symmetry of NoC components, the models built are very 
accurate (with maximum and mean error of less than $7 \%$ and $5 \%$, respectively) when compared to the actual values. In the $\times$ pipes architecture, each core is connected to a separate NI [36]. Hence, we consider the power consumption of the NI to be part of the power consumption of the core.

The impact of the targeted frequency of operation on the area and energy consumption of an example $5 \times 5$ switch obtained from layout-level estimates is presented in Figure 8. Note that we plot the energy values (in power $/ \mathrm{MHz}$ ) instead of the total power. This is to avoid the inherent increase in power consumption due to increase in frequency of the network. When the targeted frequency of operation is below a certain frequency, referred to as the nominal operating frequency (around $250 \mathrm{MHz}$ in the plots), the area and energy values for the switch remains the same. However, as the targeted frequency increases beyond the nominal frequency, the area and energy values start increasing linearly with frequency. This is because, the synthesis tool (such as Synopsys DC [34]) tries to match the desired high operating frequency by utilizing faster components that have large area and energy overhead. When performing the area, power estimates, we also model this impact of desired operating frequency on the switch area, power consumption.

\section{Experimental Results}

In this section, we present detailed experimental studies of our approach (which we further refer to as INT-TOP meaning message-dependent deadlock avoidance integrated with topology synthesis process) and compare it with traditional approaches:

(1) Using logically separate networks $(L-S E P)$ : In this scheme, we use separate buffers at each input, with as many buffers as the different message types, modeling the virtual channel based approach to remove message-dependent deadlocks.

(2) Using physically separate networks $(P-S E P)$ : In this scheme, we design physically different networks for each message type. For both these schemes we apply our topology synthesis procedure to obtain the network topologies.

(3) With a design that has no support to avoid message-dependent deadlocks $(O R I G)$. Note that this base system cannot be employed in SoCs, as it cannot guarantee proper system operation. We present the experimental results for this scheme to only evaluate the overhead incurred in the other schemes to support deadlock-free operation.

\subsection{Comparison on SoC designs}

We apply the deadlock prevention methods to five different SoC designs: Multimedia system (MULT 30 cores), IMage Processing application (IMP-27 cores), Video PROCessor (VPROC-42 cores), MPEG4 decoder (12 cores) and Video Object Plane Decoder (VOPD-12 cores). The communication characteristics of some of these benchmarks is presented in [40]. There are two types of messages 


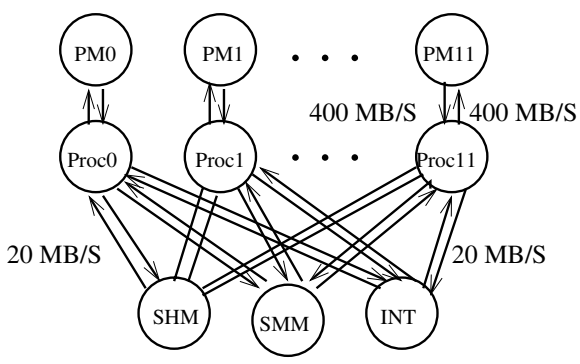

(a) Core graph

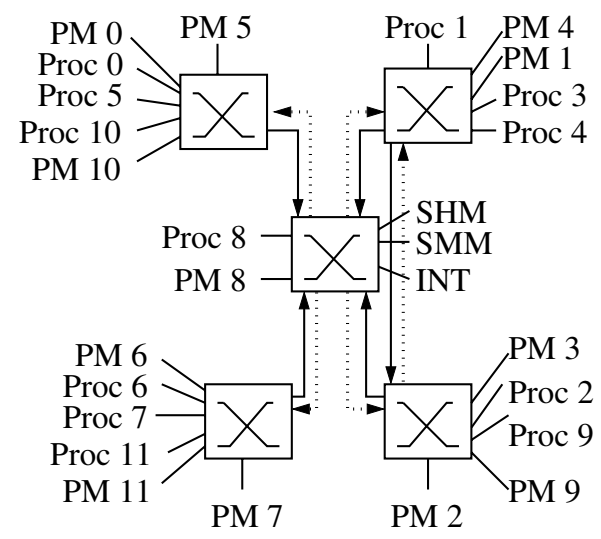

(b) Designed topology

Fig. 9. Core graph and designed topology for IMP

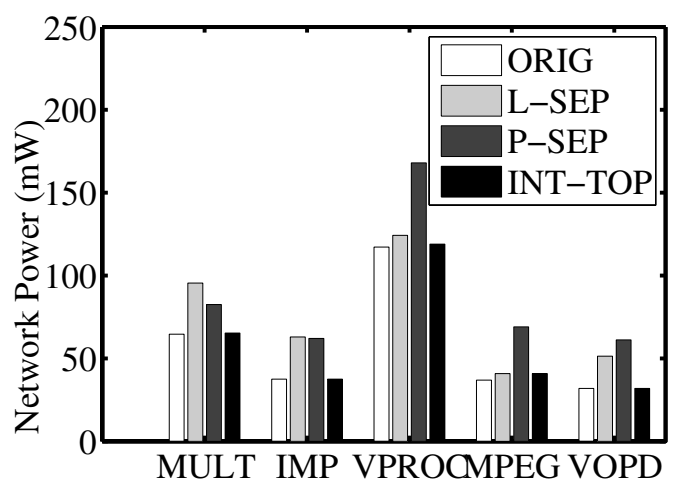

Fig. 10. Power consumption of different schemes

that are supported in each design: request and response. Each design consists of almost equal number of request and response traffic flows. This is because, every processor core communicates through the memory core, necessitating twoway communication (hence a request and response traffic flow) between the processors and memories. To make a fair comparison of the different schemes, we use the same synthesis approach and design constraints for synthesizing the topologies.

The communication pattern (core graph) for one of the applications (IMP) and the best synthesized topology for our proposed scheme (INT-TOP) are presented in Figures 9(a) and 9(b). The design consists of 12 processors (Proc 0 to Proc 11), a private memory for each processor (PM 0 to PM 11), a shared memory (SHM), a semaphore memory (SMM) and an interrupt device (INT). In the application, all communication from the processors are of request message type 
and communication to the processors are of response message type. In Figure $9(\mathrm{~b})$, those links that support request message type are in bold and those links that support response message type are dashed.

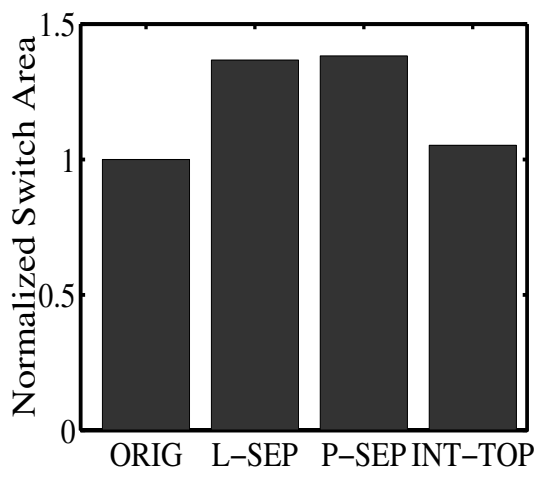

Fig. 11. Normalized switch area for the different schemes

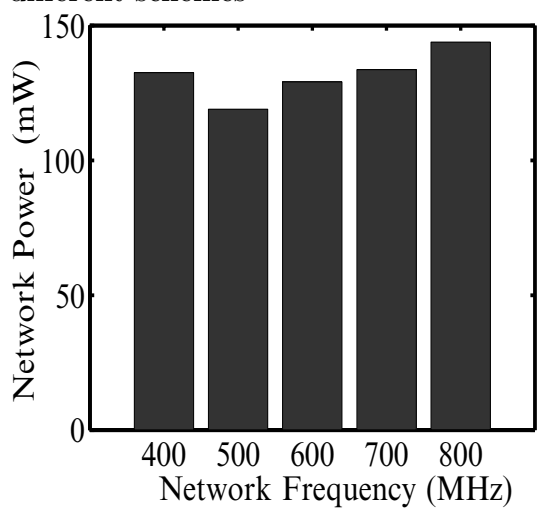

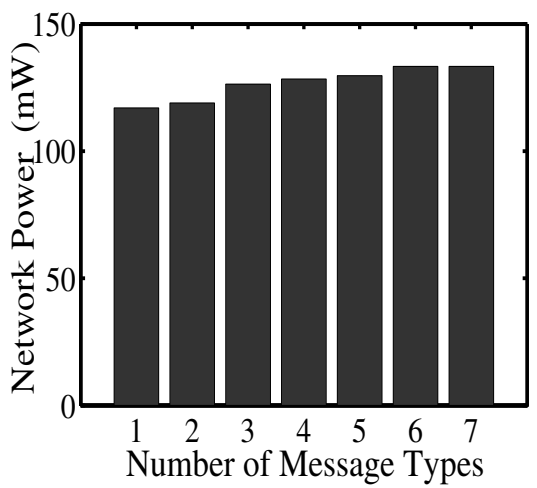

Fig. 12. Effect of number of message types

Fig. 13. Power consumption variation with frequency

The network power consumption, based on the functional traffic for the various designs using the different schemes is presented in Figure 10. As seen from this figure, the INT-TOP scheme presented in this work, outperforms the two conventional message-dependent deadlock avoidance schemes: $L-S E P$ and $P$-SEP. Our proposed scheme leads to an average of $38.5 \%$ reduction in NoC power consumption when compared to the state-of-the-art deadlock avoidance schemes. When compared to our INT-TOP scheme, the $L$-SEP scheme requires large buffering requirements, as each virtual channel needs separate buffering resources. The $P$-SEP scheme requires more switches than the INT-TOP scheme, 
as the request and response messages utilize different networks. Interestingly, our proposed scheme incurs only a $2.5 \%$ increase in power consumption when compared to the ORIG scheme, where no message-dependent deadlock avoidance support is provided. This is mostly due to the efficient allocation of links to the different message types by our topology synthesis procedure. The switch area for the different schemes for the SoC designs, normalized with respect to the area of the base system $(O R I G)$ is presented in Figure 11. The proposed method results in an average of $30.68 \%$ reduction in area when compared to the state-of-the-art schemes.

\subsection{Effect of Different Number of Message Types}

In this sub-section, we examine the power consumption of the proposed scheme, when the number of different message types is varied. The number of message types in a system depends on the underlying computation architecture. Cache coherent systems typically support several different message types. As an example, the S-1 multi-processor supports 4 different message types [18] and each type must be mapped onto different resources in the network. In [17], a more sophisticated protocol is used, which leads to seven different message types. To see the impact on the number of different message types, we created a synthetic benchmark having the traffic characteristics of the $V P R O C$ design. In this benchmark, around 80 different traffic flows exist, each one representing a message. We fixed the number of messages and varied the number of message types in the design from 1 to 7 . The network power consumption for our proposed scheme, for the different number of message types is presented in Figure 12. This figure shows that our proposed scheme results in efficient designs, even for a large number of message types. Moreover, the rise in power consumption with an increasing number of message types saturates (designs with 6 and 7 message types have nearly the same power consumption), as most messages are already mapped onto unique links in the network.

\subsection{Frequency Trade-offs}

The algorithm presented here can also be used to perform frequency selection for a certain design. In this case, the frequency of operation of the NoC can be varied and the best topology can be synthesized for each frequency point. A higher operating frequency results in links having more bandwidth. Thus a smaller NoC can satisfy the design constraints. A trade-off curve for frequency vs power consumption of the network for the VPROC is presented in Figure 13. From such a curve, the most power-efficient operating frequency can be chosen for the design.

\section{Conclusions}

For Networks on Chips (NoCs) to be used in industrial designs, NoCs should guarantee proper system operation under all conditions. Achieving deadlock-free 
operation of the network with minimum power consumption and area overhead is critical for application-specific NoCs. In this work, we have focused on addressing the major issue of avoiding routing and message-dependent deadlocks during the network operation. We have shown that by mapping the different message types onto different network resources during the topology mapping and synthesis phase, we can achieve large reductions in network power consumption and network area when compared to the state-of-the-art approaches. In future work, we plan to compare deadlock recovery schemes with the proposed scheme for NoCs.

\section{Acknowledgments}

This work is supported by the US National Science Foundation (NSF, contract CCR-0305718) for Stanford University. It is also supported by the Swiss National Science Foundation (FNS, Grant 20021-109450/1) and the Spanish Government Research Grant TIN2005-5619. The work is also supported by a grant from Semiconductor Research Corporation (SRC project number 1188) and a grant by STMicroelectronics for DEIS.

\section{References}

1. L. Benini and G.De Micheli, "Networks on Chips: A New SoC Paradigm", IEEE Computers, pp. 70-78, Jan. 2002.

2. M. Sgroi et al., "Addressing the System-on-a-Chip Interconnect Woes Through Communication-Based Design", Proc. DAC, pp. 667-672, June 2001.

3. S. Kumar et al., "A Network on Chip Architecture and Design Methodology", Proc. ISVLSI, pp. 117-122, April 2002

4. P. Guerrier, A. Greiner, "A generic architecture for on-chip packet-switched interconnections", Proc. DATE, pp. 250-256, March 2000.

5. K. Goossens et al., "The Aethereal network on chip: Concepts, architectures, and implementations", IEEE Design and Test of Computers, Vol. 22(5), pp. 21-31, SeptOct 2005 .

6. W. Dally, B. Towles, "Route Packets, not Wires: On-Chip Interconnection Networks", Proc. DAC, pp. 684-689, June 2001.

7. Y. H. Song, T. M. Pinkston, "A Progressive Approach to Handling MessageDependent Deadlock in Parallel Computer Systems", IEEE TPDS, Vol. 14(3), pp. 259-275, March 2003.

8. Y. Choi, "Deadlock Recovery Based Router Architectures for High Performance Networks", PhD Dissertation, University of Southern California, June 2001.

9. G. Chiu, "The Odd-Even Turn Model for Adaptive Routing", IEEE TPDS, Vol. 11(7), pp. 729-738, July 2000.

10. C. Glass, L. Ni, "The turn model for adaptive routing", Proc. ISCA, pp. 278-287, 1992.

11. J. Duato, "A New Theory of Deadlock-Free Adaptive Routing in Wormhole Networks", IEEE TPDS, Vol. 8(8), pp. 790-802, Aug 1997.

12. D. Starobinksi et al., "Application of network calculus to general topologies using turn-prohibition", IEEE/ACM Transactions on Networking, Vol. 11, Issue 3, pp. 411-421, June 2003. 
13. W. J. Dally, H. Aoki, "Deadlock-Free Adaptive Routing in Multi-computer Networks Using Virtual Channels", IEEE TPDS, Vol. 4(4), pp. 466-475, April 1993.

14. S. Scott, G. Thorson, Optimized Routing in the Cray T3D", Proc. Workshop Parallel Computer Routing and Comm., pp. 281-294, May 1994.

15. S. Scott, G. Thorson, "The Cray T3E Network: Adaptive Routing in a High Performance 3D Torus", Proc. Symp. Hot Interconnects IV, pp. 147-156, Aug. 1996.

16. J. Carbonaro, Cavallino, "The Teraflops Router and NIC", Proc. Symp. Hot Interconnects IV, pp. 157-160, Aug. 1996.

17. S.S. Mukherjee et al., "The Alpha 21364 Network Architecture", Proc. Symp. HOT Interconnects 9, pp. 113-117, Aug. 2001.

18. L. Widdoes, S. Correll, :The S-1 Project: Developing High Performance Computers", Proc. COMPCON, pp. 282-291, Spring 1980.

19. "http://www.st.com".

20. J. Laudon, D. Lenoski," The SGI Origin: A ccNUMA Highly Scalable Server", Proc. ISCA, pp. 241-251, June 1997.

21. D. Lenoski et al., " The Directory-Based Cache Coherence Protocol for the DASH Multiprocessor", Proc. ISCA, pp. 148-159, 1990.

22. A. Hansson, K. Goossens, A. Radulescu, "UMARS: A Unified Approach to Mapping and Routing on a Combined Guaranteed Service and Best-Effort Network-onChip Architecture", Technical Report 2005/00340, Philips Research, April 2005.

23. B. Gebremichael et al., "Deadlock Prevention in the Aethereal Protocol", Proc. Working Conference on Correct Hardware Design and Verification Methods (CHARME), Oct 2005.

24. J. Hu, R. Marculescu, 'Exploiting the Routing Flexibility for Energy/Performance Aware Mapping of Regular NoC Architectures', Proc. DATE, March 2003.

25. S. Murali, G. De Micheli, "SUNMAP: A Tool for Automatic Topology Selection and Generation for NoCs", Proc. DAC 2004.

26. S. Murali et al., "Mapping and Physical Planning of Networks on Chip Architectures with Quality-of-Service Guarantees", Proc. ASPDAC 2005.

27. A.Pinto et al., "Efficient Synthesis of Networks on Chip", ICCD 2003, pp. 146-150, Oct 2003.

28. W.H.Ho, T.M.Pinkston, "A Methodology for Designing Efficient On-Chip Interconnects on Well-Behaved Communication Patterns", HPCA 2003, pp. 377-388, Feb 2003.

29. T. Ahonen et al. "Topology Optimization for Application Specific Networks on Chip", Proc. SLIP 04.

30. K. Srinivasan et al., "An Automated Technique for Topology and Route Generation of Application Specific On-Chip Interconnection Networks", Proc. ICCAD '05.

31. A. Hansson et al., "A unified approach to constrained mapping and routing on network-on-chip architectures", pp. 75-80, Proc. ISSS 2005.

32. S. Murali et al., "Designing Application-Specific Networks on Chips using Floorplan Information", Proc. ICCAD 2006.

33. W. J. Dally, B. Towles, "Principles and Practices of Interconnection Networks", Morgan Kaufmann, Dec 2003.

34. "http://www.synopsys.com".

35. "http://www.cadence.com".

36. S. Stergiou et al., " $\times$ pipesLite: a Synthesis Oriented Design Library for Networks on Chips", pp. 1188-1193, Proc. DATE 2005.

37. F. Angiolini et al., "Contrasting a NoC and a Traditional Interconnect Fabric with Layout Awareness", pp. 124-129, Proc. DATE 2006. 
38. A. Pullini, F. Angiolini, D. Bertozzi, L. Benini, "Fault Tolerance Overhead in Network-on-Chip Flow Control Schemes", Proc. SBCCI, pp. 224-229, 2005.

39. www.ocpip.org

40. D. Bertozzi et al., "NoC Synthesis Flow for Customized Domain Specific MultiProcessor Systems-on-Chip", IEEE Transactions on Parallel and Distributed Systems, Feb 2005. 